\title{
Effect of Gamma Irradiation on the Mechanical Properties and Thermal Properties of Poly(3-Hydroxybutyrate-Co-3-hydroxyhexanoat e)(PHBHHx) Based Films
}

\author{
Yang LIU*, Yu ZHAI, Wei CAO, Bolong LI, Liancai WANG, Xinmiao ZENG \\ Beijing Key Laboratory of Radiation Advanced Materials, Beijing Research Center for \\ Radiation Application, Beijing 100015, China
}

\begin{abstract}
Poly(3-hydroxybutyrate-co-3-hydroxyhexanoate) (PHBHHx) is a naturally sourced polymer with good biocompatibility and potential applications in biomedical field after sterilization. Radiation sterilization technology has been extensively applied on medical products. However, for polymer-based biomedical materials, gamma irradiation can cause random chain scission on the polymer backbone. In this paper, we studied the effect of gamma irradiation on the mechanical and thermal properties of PHBHHx. The tensile strength and fracture strain of PHBHHx decreased after irradiation, while the brittleness increased after irradiation. Thermogravimetric analysis (TGA) and differential scanning calorimetry (DSC) results showed that the melting temperatures of irradiated PHBHHx are lower than the non-irradiated films, but the irradiated specimen shows a higher decomposition temperature than non-irradiated PHBHHx.
\end{abstract}

\section{Introduction}

Polyhydoxyalkanoates (PHAs) are polyesters produced by microorganisms under unbalanced growth conditions. The polymers are generally biodegradable and have good biocompatibility, which makes them attractive for biomedical applications. Several PHAs have been applied in biomedical field as implant materials, including poly 3-hydroxybutyrate (PHB), poly(hydroxybutyrate-co-hydroxyvalerate) (PHBV), poly

\footnotetext{
* Corresponding author: yangyang7803@bit.edu.cn
} 
4-hydroxybutyrate (P4HB), poly(3-hydroxyoctanoate) (PHO), and PHBHHx. PHBHHx was reported to have better performance over all other PHAs as mentioned above.

Biological materials must be sterilized before use. The traditional sterilization method, such as gas sterilization with ethylene oxide and steam sterilization, are not suitable for PHBHHx-based films. Gamma irradiation is the standard method in the medical device industry as it is cost-effective, and can be applied at room temperature.

However, gamma irradiation can cause random chain scission or chain crosslinking on the polymer backbone. The aim of this study is to investigate the effect of gamma irradiation on the properties of PHBHHx by thermal and mechanical analysis.

\section{Experimental}

\subsection{Materials}

PHBHHx containing $12 \%$ hydroxyhexanoate was kindly donated by department of biological science and biotechnology of Tsinghua University (produced by Shantou Lianyi Biotech Co., Guangdong, China). PHBHHx was purified before use: PHBHHx was dissolved in chloroform under vigorous agitation for $2 \mathrm{~h}$ at $65{ }^{\circ} \mathrm{C}$, then precipitated in methanol, and finally dried under vaccum. Purified PHBHHx is a white powder. All other solvents were of analytical grade.

\subsection{Film preparation and irradiation}

Sample films were prepared by solution casting method. All samples were irradiated in sealed packaging using a ${ }^{60} \mathrm{Co}$ source at the Department of Applied Chemistry of Peking University at room temperature, and the dose was 25,45 , and $70 \mathrm{kGy}$.

\subsection{Methods}

The spectra were obtained with an accumulation of 100 scans and with a resolution of 4 $\mathrm{cm}^{-1}$. The tensile properties of PHBHHx films were measured by a SANS universal testing machine with a loadcell of $100 \mathrm{~N}$ with a strain rate of $20 \mathrm{~mm} / \mathrm{min}$. The differential scanning calorimetry (DSC) studies were carried out at heating ramp $10{ }^{\circ} \mathrm{C} \min ^{-1}$ from $0{ }^{\circ} \mathrm{C}$ to $150{ }^{\circ} \mathrm{C}$ using a Mettler-Toledo DSC 1. Thermogravimetric analysis (TGA) on PHBHHx films were performed with Shimadzu TGA-50 thermal analyzer under a nitrogen atmosphere with a heating speed of $10^{\circ} \mathrm{C} \cdot \mathrm{min}^{-1}$. 


\section{Results and discussion}

\subsection{Infrared spectroscopy}

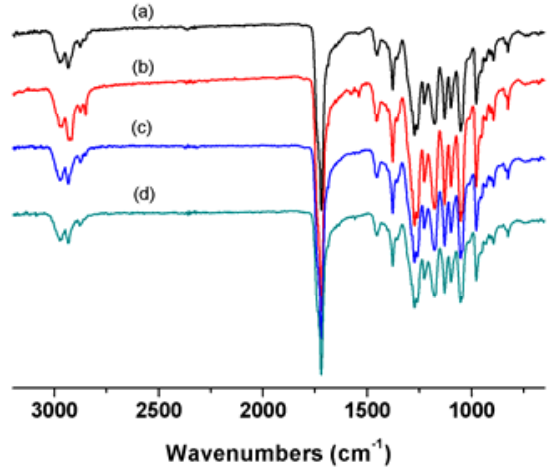

Fig. 1 ATR-FTIR spectra of control PHBHHx and irradiated PHBHHx: (a) control PHBHHx, (b) irradiated with $25 \mathrm{kGy}$ dose, (c) irradiated with $45 \mathrm{kGy}$ dose, (d) irradiated with $70 \mathrm{kGy}$ dose.

Fig. 1 shows the ATR-FTIR spectras of control PHBHHx and irradiated PHBHHx films.. All infrared absorption peak of irradiated specimens can be found in control specimen spectra, suggesting that there is no significant change between the control and the irradiated specimens. The absorption peaks of $2976 \mathrm{~cm}^{-1}, 2933 \mathrm{~cm}^{-1}, 2875 \mathrm{~cm}^{-1}$, is the stretching of $\mathrm{C}-\mathrm{H}$ bond. The results indicate that there is no major change of chemical structure between non-irradiated PHBHHx and PHBHHx samples irradiated with either $25 \mathrm{kGy}, 45 \mathrm{kGy}$, or $70 \mathrm{kGy}$ dose.

\subsection{Mechanical behavior}

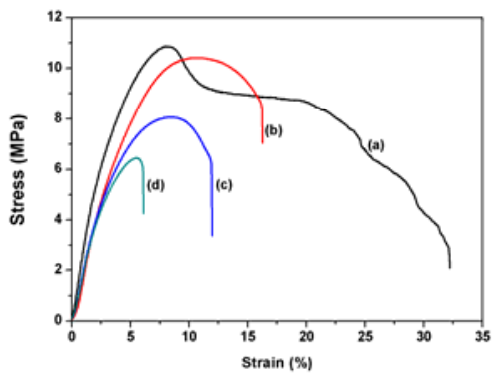

Fig. 2 The tensile stress versus strain plots of control and irradiated PHBHHx: (a) control, (b) irradiated with 25 kGy dose, (c) irradiated with 45 kGy dose, (d) irradiated with 70 kGy dose. 
.Tensile testing is very useful to determine how gamma irradiation affects the mechanical properties of PHBHHx films. Fig. 2 shows the typical stress-strain plots of control and irradiated PHBHHx films. Mechanical properties are generally characterized by two parameters, the tensile strength and the elongation at break. Table 1 summarizes the two parameters of the control and irradiated specimens. As shown in Fig. 2 and Table 1, the elongation at break reduced from $49.13 \%$ for non-irradiated PHBHHx to $19.11 \%, 11.89 \%$, and $6.14 \%$ for PHBHHx irradiated with $25 \mathrm{kGy}, 45 \mathrm{kGy}$, and $70 \mathrm{kGy}$, respectively. The elongation at break of the irradiated PHBHHx with $70 \mathrm{kGy}$ is even lower than $10 \%$, which can be classified as brittle. The tensile strength decreased from 8.76 for non-irradiated PHBHHx to 7.10 for irradiated one with the increase of irradiation dose, except for the PHBHHx irradiated with $25 \mathrm{kGy}$. We assume that significant chain scission took place in the amorphous regions of the irradiated PHBHHx samples, which resulted in a dramatic decrease in molecular weight, leading to less chain entanglements in amorphous regions. As a result, the tensile strength of the irradiated PHBHHx samples were slightly lower than those non-irradiated PHBHHx sample.

Table 1 Mechanical Properties Of Control Phbhhx And Irradiated Phbhhx

\begin{tabular}{|c|c|c|}
\hline Dose $(\mathrm{kGy})$ & tensile strength $(\mathrm{MPa})$ & elongation at break (\%) \\
\hline 0 & 8.76 & 49.13 \\
\hline 25 & 10.18 & 19.11 \\
\hline 45 & 8.11 & 11.89 \\
\hline 70 & 7.10 & 6.14 \\
\hline
\end{tabular}

\subsection{Thermal behavior}

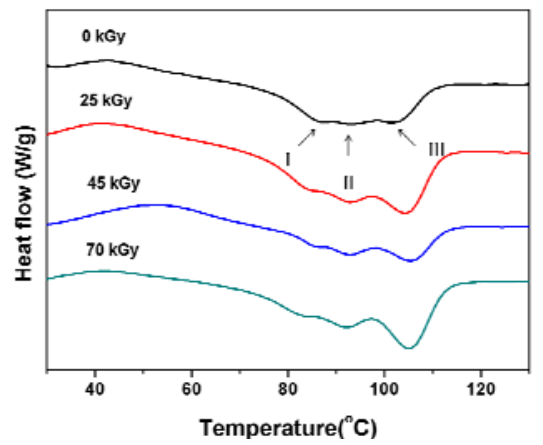

Fig. 3 Melting curves for control PHBHHx and irradiated PHBHHx. Peaks I, II, and III denote the low-, middleand high-temperature melting, respectively. 
Three melting peaks were observed in the DSC heating cruves for control and irradiated specimens as reported (Fig. 3), the occurrence of peak I was a result of the melting of crystals formed upon long-time annealing, the other two main melting edothermic peaks II and III are caused by the model of melting, recrystallization and remelting of PHB crystals during the heating process. With the increase of irradiated dose, the melting temperature of peak I shifted towards a lower temperaturę. A similar shift was also observed in peak II, which may due to the decrease of PHBHHx crystal size.
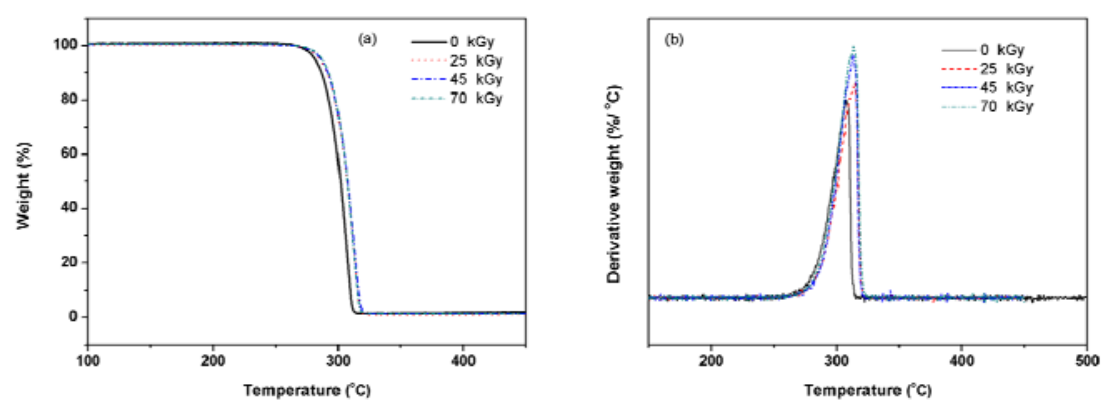

Fig. 4 TGA (a) and DTG (b) curves of. control and irradiated PHBHHx to the indicated doses.

Thermogravimetric analysis (TGA) and differential thermogravimetric analysis (DTG) curves are shown in Fig. 4. The TGA curves of irradiated specimens shifted towards a higher temperature compared to control specimen (Fig. 4a), the initial decomposition temperature of PHBHHx irradiated with $70 \mathrm{kGy}$ located at $287.16^{\circ} \mathrm{C}$, which is $6{ }^{\circ} \mathrm{C}$ higher than that of control specimen $\left(281.28{ }^{\circ} \mathrm{C}\right)$. The derivation of the thermal decomposition curve (Fig. 4b) shows the difference of peak temperatures between the irradiated and non-irradiated samples. The temperature of total weight loss of the control PHBHHx sample was $308.45^{\circ} \mathrm{C}$, and the value of irradiated PHBHHx with $25 \mathrm{kGy}, 45 \mathrm{kGy}$, and 70 kGy are $314.70{ }^{\circ} \mathrm{C}, 314.05{ }^{\circ} \mathrm{C}$, and $313.93{ }^{\circ} \mathrm{C}$, respectively. We also notice that the irradiated specimen shows a slightly higher decomposition temperature than the control PHBHHx, indicating that the scissions that occur due to gamma irradiation do not affect the thermal decomposition temperature of the sample.

\section{Conclusion}

We have studied the effect of gamma irradiation on the structure and properties of PHBHHx films. The mechanical studies showed that tensile strength and fracture strain of PHBHHx decreased after irradiation with the increase of brittleness.

Thermogravimetric analysis and differential scanning calorimetry results showed that the melting temperature $\left(T_{\mathrm{m}}\right)$ of PHBHHx films decreased with the increase of irradiation dose, 
due to the decrease of PHBHHx crystal size.

\section{Acknowledgements}

The authors greatly acknowledge the support of the Beijing Key Laboratory of Radiation Advanced Materials of Beijing Research Center for Radiation Application.

\section{References}

1. Mitomo, H., Watanabe, Y., Yoshii, F. \& Makuuchi, K. (1995). Radiat Phys Chem 46, 233-238.

2. Bahari, K., Mitomo, H., Enjoji, T., Hasegawa, S., Yoshii, F. \& Makuuchi, K. (1997). Angew Makromol Chem 250, 31-44.

3. Hermida, E. B., Mega, V. I., Yashchuk, O., Fernandez, V., Eisenberg, P. \& Miyazaki, S. S. (2008). Macromol Symp 263, 102-113.

4. Miyazaki, S. S., Yep, A. R., Kolton, F., Hermida, E. B., Povolo, F., Fernandes, E. G. \& Chiellini, E. (2003). Macromol Symp 197, 57-64.

5. Oliveira, L. M., Araujo, E. S. \& Guedes, S. M. L. (2006). Polym Degrad Stabil 91, 2157-2162.

6. Rosario, F., Corradini, E., Casarin, S. A. \& Agnelli, J. A. M. (2013). Journal of Polymers and the Environment 21, 789-794.

7. Oliveira, L. M., Araujo, P. L. B. \& Araujo, E. S. (2013). Mater Res-Ibero-Am J 16, 195-203.

8. Luo, S. \& Netravali, A. N. (1999). J Appl Polym Sci 73, 1059-1067. 